\title{
EFEITO DE INGREDIENTES NA QUALIDADE DA MASSA DE PÃO DE FORMA CONGELADA NÃO FERMENTADA DURANTE O ARMAZENAMENTO
}

\author{
Luiz C. GUTKOSKI ", Cibele M. BREHM , Elisa dos SANTOS ', Natália MEZZOMO
}

\section{RESUMO}

O objetivo do trabalho foi estudar o efeito combinado de fermento instantâneo, lecitina de soja e extrato de soja, na produção de massa de pão congelada não fermentada, durante o período de sessenta dias de armazenamento da massa. O estudo de formulação foi realizado na padaria experimental do Centro de Pesquisa em Alimentação da Universidade de Passo Fundo, utilizando três níveis de fermento instantâneo $(0,8 \%, 1,3 \%$ e $1,8 \%)$, três níveis de lecitina de soja $(0,5 \%, 1,5 \%$ e $2,5 \%)$ e três níveis de extrato de soja $(1 \%, 3 \%$ e $5 \%)$, totalizando 15 tratamentos, em fatorial incompleto. A massa foi dividida em porções de $150 \mathrm{~g}$, modelada e congelada em ultracongelador marca Klimaquip, na velocidade do ar de $1,23^{\circ} \mathrm{C}$ min e circulação do ar a $5 \mathrm{~ms}$. $\mathrm{O}$ descongelamento das massas foi realizado aos 30 dias e aos 60 dias de armazenamento, fermentação em câmara regulada na temperatura de $32 \pm 1^{\circ} \mathrm{C}$ e na umidade relativa de $80 \%$. O cozimento foi realizado em forno rotativo de laboratório, regulado na temperatura de $190^{\circ} \mathrm{C}$ por 18 minutos. Variáveis fisicas e funcionais foram monitoradas e a metodologia de superficie de resposta usada para otimizar o estudo de formulação de pão de forma. Entre os ingredientes estudados na formulação de pão de forma, somente as concentrações de fermento instantâneo e de extrato de soja interferiram significativamente nas variáveis respostas. O volume e a produção de gás aumentaram com o tempo de fermentação da massa sendo proporcionais à concentração de fermento utilizado na formulação. O volume específico, a cor da crosta e o escore de pontos dos pães aumentaram com a elevação da concentração de fermento instantâneo e redução do teor de extrato de soja. Com o aumento do tempo de armazenamento de 30 dias para 60 dias ocorreu uma perda nas características de qualidade dos pães, exceto para volume específico que se manteve estável.

Palavras-chave: trigo; farinha; congelamento; fermento; lecitina; extrato de soja.

\section{SUMMARY}

EFFECT OF INGREDIENTS ON THE QUALITY OF NON-FERMENTED FROZEN FORM BREAD DOUGH DURING STORAGE. The objective of this work is to study the combined effect of instant yeast, soy lecithin and soy extract in the production of nonfermented frozen form bread during sixty days of dough storage. The procedures were carried out in the bakery of the Center of Research in Feeding of the University of Passo Fundo, using three levels of instant yeast (0,8\%, 1,3\% e 1,8\%), three levels of soy lecithin $(0,5 \%, 1,5 \%$ e $2,5 \%)$ and three levels of soy extract $(1 \%, 3 \%$ e $5 \%)$, completing 15 treatments, in incomplete factorial. The dough was divided in $150 \mathrm{~g}$ pieces, shaped and frozen in the ultra freezer, mark Klimaquip, in the speed of $1,23^{\circ} \mathrm{C}$ min and $5 \mathrm{~ms}$ air circulation. The samples were thawed after 30 days and 60 days of storage, the fermentation was in a fermentation cabinet at $32 \pm 1^{\circ} \mathrm{C}$ and in relative humidity of $80 \%$ and cooking was done in a laboratory rotative oven, at $190^{\circ} \mathrm{C}$ for 18 minutes. Functional and physics variables were monitored and answer surface methodology was used to optimize the formulation study of the form bread. Among the ingredients studied in the form bread formulation, only instant yeast and soy extract concentrations had significant interferences in answer variables. The volume and the gas production increased with the dough fermentation in the same proportion as yeast concentration used in the formulation. The form bread specific volume, crust color and points score increased with the instant yeast concentration raising and the decrease of soy extract. With the storage time increasing from 30 days to 60 days, there was a quality loss in the bread characteristics, except for the specific volume that remained stable.

Keywords: wheat; flour; freezing; yeast; lecithin; soy extract.

\section{1 - INTRODUÇÃO}

A evolução tecnológica do pão se deve fundamentalmente ao seu grande consumo. A sua importância na alimentação somente é reconhecida quando ocorre a falta do produto na mesa do consumidor, embora em algumas regiões do mundo tenha ocorrido uma retração no consumo [18]. Pão de forma é o produto obtido pela cocção da massa em formas, apresentando miolo elástico e homogêneo, com poros finos e casca fina e macia [4], sendo produzido a partir da mistura dos ingredientes farinha, água, fermento e sal [27], divisão, modelagem, fermentação e cozimento. Devido a sua alta atividade de água, o pe-

Recebido para publicação em02/03/2004. Aceito para publicação em 11/08/2005(001301).

Faculdade de Agronomia e Medicina Veterinária, Universidade de Passo Fundo. CP: 611. CEP: 99001-970, Passo Fundo-RS. E-mail: gutkoski@upf.br

Centro de Pesquisa em Alimentação, Universidade de Passo Fundo. A quem a correspondência deve ser enviada. ríodo de comercialização é relativamente curto, pois se trata de um alimento perecível.

Um dos fatores que limita a vida-de-prateleira do pão é o envelhecimento que ocorre devido à retrogradação e que contribui para aumentar a firmeza do miolo, dando uma sensação de produto seco ao ser ingerido [26]. Esses fatores, aliados à necessidade de diminuir os custos operacionais e expandir o mercado, têm levado panificadores a exigirem novas tecnologias e o desenvolvimento de novos métodos de produção de massas [2].

O congelamento é um dos melhores métodos de preservação conhecido e é empregado para retardar alterações na qualidade dos alimentos. Por esta razão, o mercado para produtos de panificação congelados tem crescido rapidamente e vem sendo pesquisado e aplicado em diversos países. No Brasil, trata-se de uma tecnologia recente e pouco estudada até o presente momento.

As primeiras tentativas de produção de pão a partir de massa congelada ocorreram em 1 926, na Áustria, pelo uso de baixas temperaturas para o retardamento da 
etapa de fermentação da massa, numa tentativa de reduzir o trabalho noturno nas padarias [24]. Na década de 50 , algumas padarias de pequeno porte consideravam conveniente e econômico o congelamento de porções reserva de massa, que podiam ser mantidas por alguns dias ou semanas no congelador, para serem então descongeladas, fermentadas e assadas nos horários de maior demanda [18]. Nos Estados Unidos, os primeiros trabalhos com massa congelada foram realizados com o emprego de misturadores de cimento e congeladores de sorvete, devido à inexistência de equipamentos apropriados [14].

O uso de massa congelada atende as exigências dos consumidores por pães sempre frescos, isto é, recémassados, permitindo ao mesmo tempo maior flexibilidade na produção [25].

A massa congelada deve resistir às condições adversas antes de ser forneada, devendo no descongelamento fermentar em um período de tempo aceitável e produzir pão com volume normal e com características sensoriais desejáveis [28].

HAVET, MANKAY \& LE BAIL [11] em estudo sobre o efeito do congelamento na qualidade de pão francês, afirmam que o volume é influenciado prioritariamente pela viabilidade das leveduras e a qualidade da rede de glúten. A levedura é afetada pelo congelamento, sendo normalmente recomendado o lento como forma de preservar a atividade das leveduras. A rede de glúten é danificada durante o congelamento, fenômeno que afeta a habilidade da massa reter o gás carbônico, minimizando o volume do pão. Os resultados do trabalho mostram que a atividade da levedura está fortemente relacionada com a velocidade de congelamento da massa. De acordo com FRANCISCHI, ORMENESE \& PIZZINATTO [8], o maior desafio na tecnologia de produção de pães de massas congeladas é manter a viabilidade das células e o poder de produção de gás carbônico da levedura congelada

Para BRUINSMA \& GIESENSCHLAG [5], a levedura é um dos ingredientes mais importantes na produção da massa congelada e, com certeza, o mais estudado. Recomenda-se a utilização de quantidade de levedura superior à empregada no processo convencional, que pode variar entre 4 e $6,5 \%$. A quantidade necessária de levedura depende do tempo de armazenamento da massa congelada, da formulação e do tempo de fermentação desejado após o descongelamento da massa. O dano experimentado pelas leveduras de panificação pode ser reduzido até certo ponto omitindo ou minimizando o período de fermentação da massa antes do congelamento e mudando os niveis dos ingredientes na formulação [22].

WANG \& PONTE [28] demonstraram que o volume do pão e a estrutura do miolo são melhorados, assim como o período de fermentação é drasticamente reduzido, com a adição de $2 \%$ de glúten vital. Glúten vital é aquele tipo de glúten que não sofreu desnaturação irreversivel e, em contato com a água, reidrata-se rapidamente, recuperando sua funcionalidade intrínseca, isto é, sua viscoe- lasticidade.

Entre as deficiências apontadas na produção de pão congelado está o baixo volume, a estrutura de miolo aberta e mais firme, o tempo de fermentação excessivamente longo, a qualidade do produto razoável por apenas algumas semanas, isto é, o potencial de panificação da massa decresce substancialmente com o tempo de armazenamento e com os ciclos de congelamento e descongelamento parciais, que podem ocorrer durante a distribuição, transporte e armazenamento do produto. No entanto, devido ao enorme avanço na qualidade dos produtos de panificação trazidos pelo aprimoramento das formulações, da tecnologia de processo e da metodologia de congelamento, assim como dos tipos de levedura e de farinha, de agentes oxidantes e condicionadores, tais desvantagens estão sendo minimizadas [29].

Em razão da importância do pão para o mercado doméstico e para as panificadoras, desenvolveu-se o presente trabalho com o objetivo de estudar o emprego de fermento, lecitina de soja e extrato integral de soja ativado na produção de pão de forma, após os períodos de trinta dias e sessenta dias de armazenamento da massa congelada não fermentada.

\section{2 - MATERIAL E MÉTODOS}

\section{1 - Material}

Foi utilizada farinha de trigo especial, adquirida em moinho da região, apresentando $13,94 \%$ de umidade, $10,66 \%$ de proteína bruta, $0,57 \%$ de cinzas, 294 segundos de número de queda. A força geral do glúten (W) determinada no alveógrafo foi $184 \times 10^{\circ} \mathrm{J}$, a extensibilidade de $80 \mathrm{~mm}$ e relação P/L 0,88. O fermento tipo instantâneo marca Bruggeman, a lecitina de soja marca Solae do Brasil, o extrato integral de soja ativado marca Olvebra Industrial S.A. e a enzima alfa-amilase fúngica (40.000SKB) marca Granotec do Brasil foram fornecidos pelas empresas. O ácido ascórbico (P.A.) utilizado foi o da marca Reagen. A gordura hidrogenada, o sal, o açúcar e o açúcar invertido foram adquiridos no comércio local.

Os testes de panificação foram realizados na padaria experimental do Centro de Pesquisa em Alimentação da Universidade de Passo Fundo. A formulação foi de acordo com GUTKOSKI \& JACOBSEN NETO [9], sendo utilizados: farinha de trigo $(100 \%)$, sal refinado $(2 \%)$, açúcar $(4 \%)$, açúcar invertido (4\%), gordura vegetal hidrogenada (5\%), ácido ascórbico $(0,01 \%)$, alfa-amilase fúngica $(0,05 \%)$ e água a $4{ }^{\circ} \mathrm{C}$, adicionada de acordo com a absorção determinada no promilógrafo. As concentrações de fermento $(0,8 \%, 1,3 \%$ e $1,8 \%)$, lecitina de soja $(0,5 \%$, $1,5 \%$ e $2,5 \%$ ) e extrato de soja ( $1 \%, 3 \%$ e $5 \%$ ) estudadas foram de acordo com o delineamento experimental proposto por BOX \& BEHNKEN [3] para três niveis e três variáveis independentes.

Os ingredientes secos foram misturados na masseira marca Suprema, em velocidade lenta por dois minutos, a seguir adicionado o restante dos ingredientes, pro- 
cedendo a mistura em velocidade rápida até o completo desenvolvimento do glúten. A massa foi retirada da masseira com temperatura não superior a $22^{\circ} \mathrm{C}$, dividida em divisora manual marca Maq-Forno, em porções de 150g, e com descanso de 5 minutos para após ser modelada em modeladora marca Maq-Forno. Depois de modelada, a massa foi congelada a $-35^{\circ} \mathrm{C}$ no ultracongelador, marca Klimaquip e modelo UK 6, até que o centro da massa atingisse $21^{\circ} \mathrm{C}$, monitorado através de um termopar digital. A velocidade de congelamento foi de $1,23^{\circ} \mathrm{C} \mathrm{min}$ e a velocidade do ar de $5 \mathrm{~ms}$ [10]. Após 24 horas, as massas congeladas foram embaladas com o uso de polietileno e mantidas na temperatura de $-18^{\circ} \mathrm{C}$ para a conservação. No descongelamento e fermentação, realizados aos 30 dias e 60 dias de armazenamento, as massas foram colocadas em formas de dimensões definidas $(390 \mathrm{~mL})$, permaneceram por 120 minutos em temperatura ambiente $\left(23^{\circ} \mathrm{C}\right)$, sendo levadas para a câmara de fermentação marca Multipão, regulada na temperatura de $32 \pm 1^{\circ} \mathrm{C}$ e umidade relativa de $80 \%$. O monitoramento foi realizado pelo uso de termo-higrômetro. Após 135 minutos de fermentação foi realizado o cozimento das massas no forno piloto marca Labor Instruments Works, modelo QA 226, regulado na temperatura de $190^{\circ} \mathrm{C}$ por 18 minutos, deixado os pães resfriar por uma hora e realizadas as análises.

\section{2 - Composição química}

Os conteúdos de umidade, cinzas e proteínas foram determinados de acordo com a AACC [1], métodos números 44-15A, 08-01 e 46-13, respectivamente, realizados em triplicata. Para a determinação de proteína bruta o teor de nitrogênio foi multiplicado pelo fator 5,7.

\section{3 - Número de queda}

O número de queda foi determinado através do aparelho Falling Number, modelo 1500 Fungal (Perten Instruments, Suiça), de acordo com o método número 56-81B da AACC [1], utilizando 7 gramas de farinha, corrigido para $14 \%$ de umidade e realizado em triplicata.

\section{4 - Alveografia}

As características viscoelásticas da farinha de trigo foram determinadas no alveógrafo marca Chopin, modelo NG (Villeneuve, La Garenne, France), utilizando o método n⿳0 54-30 da AACC [1], através da pesagem de 250 gramas de farinha e volume de $129,4 \mathrm{~mL}$ de solução salina $2,5 \%$, corrigido na base de $15 \%$ de umidade. Os parâmetros obtidos nos alveogramas são tenacidade (P), que mede a sobrepressão máxima exercida na expansão da massa ( $\mathrm{mm}$ ); extensibilidade (L), que mede o comprimento da curva $(\mathrm{mm})$ e energia de deformação da massa (W), que corresponde ao trabalho mecânico necessário para expandir a bolha até a ruptura, expressa em $10 \mathrm{~J}$.

\section{5 - Volume da massa durante a fermentação}

Porções de massa $(50 \mathrm{~g})$ foram colocadas em proveta esterilizada de $1000 \mathrm{~mL}$ e sobre a porção um peso de $3,3 \mathrm{~g}$, em uma câmara de fermentação regulada na temperatura de $32^{\circ} \mathrm{C}$ e $80 \%$ de umidade relativa do ar, realizado de acordo ROUILLÉ, LE BAIL \& COURCOUX [21]. O aumento de volume após os tempos 60 minutos, 120 minutos e 180 minutos foi considerado como volume da massa, expresso em $\mathrm{mL}$.

\section{6 - Produção de gás durante a fermentação}

Porções de massa $(8 \mathrm{~g})$ foram colocadas em erlenmeyer de $300 \mathrm{~mL}$, adicionado $200 \mathrm{~mL}$ de água destilada, vedado com rolha de borracha e o frasco acoplado a um tubo graduado de $50 \mathrm{~mL}$ com solução ácida 0,00001 N de $\mathrm{HCl}$. O frasco foi colocado em uma câmara de fermentação regulada na temperatura de $32^{\circ} \mathrm{C}$ e $80 \%$ de umidade relativa do ar, realizado de acordo ROUILLÉ, LE BAIL \& COURCOUX [21]. O deslocamento do líquido no tubo correspondeu à produção de gás durante a fermentação, determinado nos tempos 60 minutos, 120 minutos e 180 minutos, expresso em $\mathrm{mL}$.

\section{7 - Avaliação dos pães}

O volume foi determinado pelo método de deslocamento de sementes de painço e o volume específico calculado pela relação entre o volume do pão assado e o seu peso, obtido pelo emprego de balança analitica. A determinação do volume específico foi realizada uma hora após o cozimento dos pães, com três repetições e os resultados expressos em $\mathrm{cm}^{3} \mathrm{~g}$.

A avaliação dos pães foi realizada segundo a planilha de pontos para pão de forma proposta por EL-DASH [6], através da análise de características externas e internas conferindo às amostras, a partir destes resultados, um valor com pontuação máxima de 100, designada como escore do pão.

\section{8 - Delineamento experimental e análise esta- tistica}

O delineamento aplicado para este estudo foi um fatorial incompleto $3^{3}$ [3], com três niveis e três fatores (fermento, lecitina de soja e extrato de soja), totalizando 15 tratamentos. Os niveis estudados foram $0,8 \%, 1,3 \%$ e $1,8 \%$ de fermento instantâneo; $0,5 \%, 1,5 \%$ e $2,5 \%$ de lecitina de soja e $1 \%$, $3 \%$ e $5 \%$ de extrato integral de soja ativado.

Para cada tratamento foram avaliados as variáveis dependentes e os dados submetidos à análise de regressão multivariada, desenvolvendo-se assim, os modelos matemáticos de segunda ordem, contendo termos lineares, quadráticos e as interações binárias das três variáveis independentes. As variáveis avaliadas foram volume da massa durante a fermentação, produção de gás durante a fermentação, cor da crosta e escore do pão. A significância do modelo foi testada pela análise de variância (teste $\mathrm{F}, \mathrm{p} \leq 0,05)$. A partir da equação de regressão múltipla foram elaborados os contornos de superficie de resposta dentro dos intervalos estudados. Para os resultados de volume da massa e de produção de gás durante a 
fermentação foi obtida a média dos dados e os gráficos plotados em duas dimensões.

\section{3 - RESULTADOS E DISCUSSÃO}

A partir dos dados experimentais das variáveis respostas estudadas e obtidos após 30 dias e 60 dias de armazenamento da massa congelada não fermentada, estabeleceram-se os modelos de regressão, em função das condições experimentais empregadas, sendo avaliada a significância dos mesmos, ao nivel de $5 \%$ de probabilidade de erro. Nos modelos completos eliminaram-se os coeficientes não significativos através do procedimento stepwise ( $p>0,10)$, obtendo-se os modelos ajustados (Tabela 1). A adequabilidade dos modelos ajustados pode ser verificada pelos coeficientes de determinação, que explicam entre 0,72 e 0,86 da variância total das respostas, e pela ausência da falta de ajuste dos resíduos. Em razão desses resultados, os modelos podem ser usados para predizer o comportamento das respostas dentro do intervalo de variação estudado.

TABELA 1 - Modelos, significância e coeficientes de determinação para as respostas $\mathrm{Y}_{2}=$ volume específico $\left(\mathrm{cm}^{3} \mathrm{~g}^{-1}\right), \mathrm{Y}_{2}=$ cor da crosta ( 0 a 10 pontos) e $Y_{1}=$ escore do pão ( 0 a 100 pontos), após 30 dias e 60 dias de armazenamento da massa congelada não fermentada

\begin{tabular}{|c|c|c|c|}
\hline Resposta & Modelo 1 & Prob $>F$ & $\mathrm{R}^{2}$ \\
\hline $\mathrm{Y}_{1-\mathrm{t} 30^{-}}$ & $-9,31+31,94 X_{1}+2,44 X_{3}-9,88 X_{1}^{2}-0,38 X_{3}^{2}-0,67 X_{1} X_{3}$ & 0,0160 & 0,75 \\
\hline$Y_{1-t 60}=$ & $-6,05+30,31 x_{1}-1,55 X_{3}-9,60 x_{1}^{2}-0,33 X_{3}^{2}-0,52 x_{1} x_{3}$ & 0,0173 & 0,74 \\
\hline $\mathrm{Y}_{2-\mathrm{t} 30}=$ & $-2,17+15,38 \mathrm{x}_{1}+0,15 \mathrm{x}_{3}-5,26 \mathrm{x}_{1}^{2}-0,26 \mathrm{X}_{3}^{2}+0,65 \mathrm{x}_{1} \mathrm{x}_{3}$ & 0,0140 & 0,85 \\
\hline$Y_{2-t 60}=$ & $-3,12+20,80 \mathrm{X}_{1}-2,93 \mathrm{X}_{3}-5,57 \mathrm{x}_{1}^{2}+0,02 \mathrm{X}_{3}^{2}+1,87 \mathrm{x}_{1} \mathrm{X}_{3}$ & 0,0249 & 0,72 \\
\hline $\mathrm{Y}_{4-\mathrm{t} 30}=$ & $-13,9+132,72 \mathrm{x}_{1}+600 \mathrm{x}_{3}-39,25 \mathrm{x}_{1}{ }^{2}-1,15 \mathrm{x}_{3}{ }^{2}-3,33 \mathrm{x}_{1} \mathrm{x}_{3}$ & 0,0010 & 0,86 \\
\hline$Y_{4-t 60}=$ & $-23,31+145,18 X_{1}-8,17 X_{3}-50,29 X_{1}^{2}+1,01 X_{3}^{2}+0,12 X_{1} X_{3}$ & 0,0027 & 0,83 \\
\hline
\end{tabular}

\section{1 - Volume específico}

O volume específico dos pães de forma elaborados a partir de massa congelada não fermentada armazenada por 30 dias e 60 dias mostrou ser dependente das concentrações de fermento instantâneo e extrato de soja adicionadas na formulação. A concentração de lecitina de soja não interferiu significativamente $(p>0,05)$ na resposta estudada. A lecitina de soja é utilizada como agente emulsificante, enquanto o extrato de soja tem como funções: aumentar a capacidade de hidratação da farinha pelo acréscimo de proteínas e branquear o miolo devido à presença das enzimas lipoxigenases [26]

Na Figura 1, representa-se na forma de gráficos de superficie de resposta, a variação do volume específico dos pães de forma em função das concentrações de fermento instantâneo e extrato de soja, nos tempos 30 dias e 60 dias de armazenamento das massas congeladas não fermentadas. O volume específico é uma característica objetiva sendo obtida pela pesagem dos pães em balança analítica, determinação do volume pelo deslocamento de sementes de painço e a obtenção do volume específico de cada pão através da relação entre seu volume e seu peso. $\mathrm{O}$ volume específico variou entre 10,52 e $18,30 \mathrm{~cm} 3 \mathrm{~g}$, e aumentou com a elevação da concentração de fermento instantâneo e diminuição do teor de extrato de soja, tanto aos 30 dias, quanto aos 60 dias de armazenamento das massas congeladas não fermentadas.

O volume específico representa, com bastante precisão, a variação de volume dos pães elaborados nos diferentes tratamentos. FERREIRA [7] afirma que, quanto maior o volume específico do pão de forma, melhor é a sua avaliação. De acordo com a planilha de pontos para pão de forma proposta por EL-DASH [6], o valor máximo obtido é 20 . O volume específico médio foi $14,69 \mathrm{~cm}^{3} \mathrm{~g}$ $14,79 \mathrm{~cm} g$ para os períodos de 30 dias e 60 dias de armazenamento da massa congelada, respectivamente. No presente trabalho, o tratamento com $1,8 \%$ de fermento, $1,5 \%$ de lecitina e $1 \%$ de extrato de soja apresentou $18,12 \mathrm{~cm}$ g de volume específico após 60 dias de armazenamento da massa congelada.

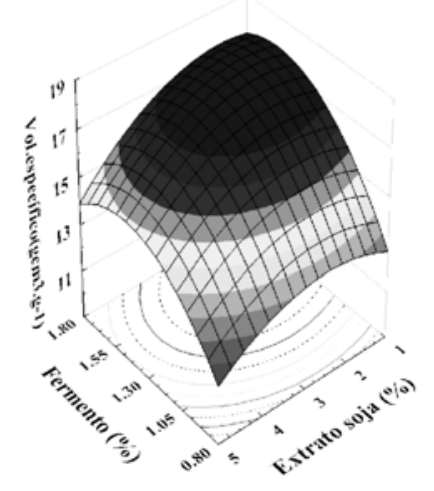

(a)

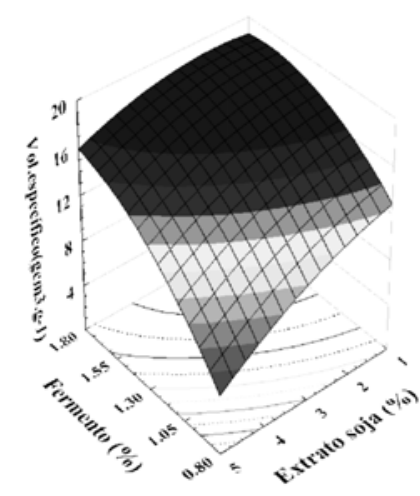

(b)

FIGURA 1 - Efeito das concentrações de fermento instantâneo e extrato de soja no volume específico (cm 'g ') de pão de forma produzido após 30 dias (a) e 60 dias (b) de armazenamento da massa congelada não fermentada 
Entre os ingredientes estudados, o fermento se mostrou mais efetivo para esta característica quando comparado com lecitina e extrato de soja. De acordo com HIRASAWA et al. [12], as leveduras de panificação são susceptiveis à danificação pelo frio durante o armazenamento da massa congelada, de tal maneira que as células de levedura não podem reter sua capacidade de produção de gás original, após o descongelamento. SALASMELLADO [23] afirma que o tipo de fermento utilizado na formulação da massa congelada influi no volume específico do pão. Pães elaborados a partir de levedura instantânea para massa doce apresentaram um volume específico médio maior que os pães produzidos a partir de levedura instantânea para massa salgada. No presente trabalho utilizou-se levedura instantânea para massa salgada e com o aumento da concentração foi verificado maior volume específico dos pães.

Outro fator importante na qualidade dos produtos elaborados com massas congeladas é a influência do tempo de armazenamento e das condições de estrutura do glúten [16]. A estrutura da matriz protéica do glúten parece ficar interrompida durante o armazenamento prolongado e repetidos ciclos de congelamento/descongelamento [28], resultando no enfraquecimento das propriedades de força da massa, perda de retenção de gás e deterioração da qualidade do produto [13].

No presente trabalho não foram observados redução no volume específico dos pães com o aumento do tempo de armazenamento das massas congeladas não fermentadas, provavelmente em função do periodo estudado. Já NEMETH, PAULLEY \& PRESTON [17] verificaram que o tempo de armazenamento da massa de pão de forma congelada não fermentada por até 200 dias, provocou uma redução no volume específico dos pães.

\section{2 - Volume da massa e produção de gás duran- te a fermentação}

Na Figura 2 está representado o efeito do tempo no aumento de volume da massa durante a fermentação realizada em câmara regulada na temperatura de $32^{\circ} \mathrm{C}$ e $80 \%$ de umidade relativa do ar, com o emprego das concentrações de $0,8 \%, 1,3 \%$ e $1,8 \%$ de fermento instantâ-

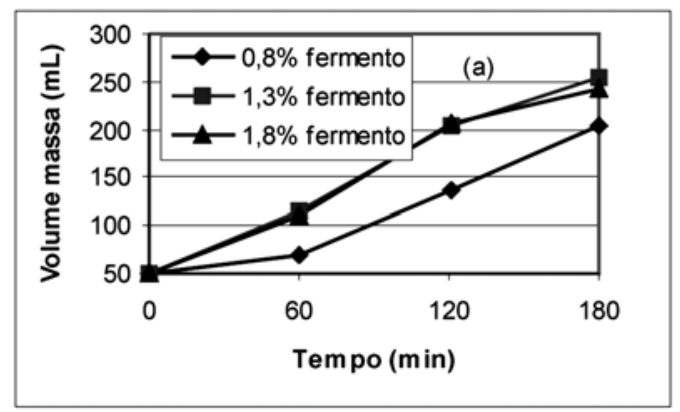

neo após 30 dias e 60 dias de armazenamento de massa congelada não fermentada. Observa-se uma elevação do volume da massa nas três concentrações de fermento estudadas, com o aumento do tempo de fermentação. Na concentração de $0,8 \%$ de fermento instantâneo, o aumento de volume foi menor quando comparado com $1,3 \%$ e $1,8 \%$. Isto refletiu em menor volume específico do pão, conforme os resultados da Figura 1.

O volume não variou quando comparados os resultados de 30 dias e de 60 dias de armazenamento das massas congeladas não fermentadas. LE BAIL et al. [15], em avaliação do volume da massa em função do tempo e condições de armazenamento, verificaram uma redução de $6,7 \%$ após 37 dias em temperatura estável de congelamento. Para as massas congeladas em temperatura flutuante, a redução de volume foi de $48 \%$, em comparação com a massa não congelada. Isto demonstra que as condições de armazenamento são mais importantes quando comparadas com o tempo para manter o volume da massa congelada. No presente trabalho a temperatura do freezer foi monitorada e mantida em valor constante, o que deve ter contribuído para a manutenção do volume da massa após o período de 60 dias de congelamento.

Na Figura 3 está representada a produção de gás carbônico em função do tempo e concentrações de fermento instantâneo após 30 dias e 60 dias de armazenamento de massa congelada não fermentada. Com o aumento da concentração de fermento instantâneo observa-se uma elevação na produção de gás. Não foi verificada redução do volume de gás carbônico após 60 dias de armazenamento da massa congelada não fermentada.

ROUILLÉ, LE BAIL \& COURCOUX [21] verificaram que o tempo de mistura interfere significativamente na produção de gás durante a fermentação de massa congelada, pois resulta em maior desenvolvimento da rede de glúten. Com o tempo de fermentação a produção de gás foi crescente, comportando-se de forma similar aos resultados obtidos no presente trabalho. Já para volume da massa, os autores observaram um platô aos 180 minutos de fermentação, indicando que, após este periodo, ocorreu a ruptura da rede de glúten, impossibilitando a retenção do gás formado durante a fermentação da massa.

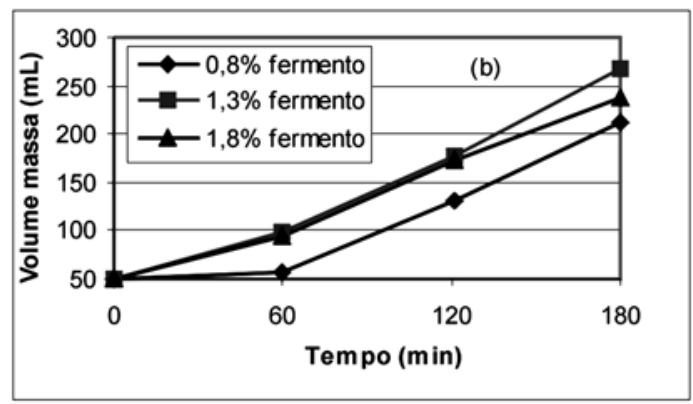

FIGURA 2 - Aumento de volume durante a fermentação da massa congelada de pão de forma nas concentrações de $0,8 \%$, 1,3\% e $1,8 \%$ de fermento após 30 dias (a) e 60 dias (b) de armazenamento 

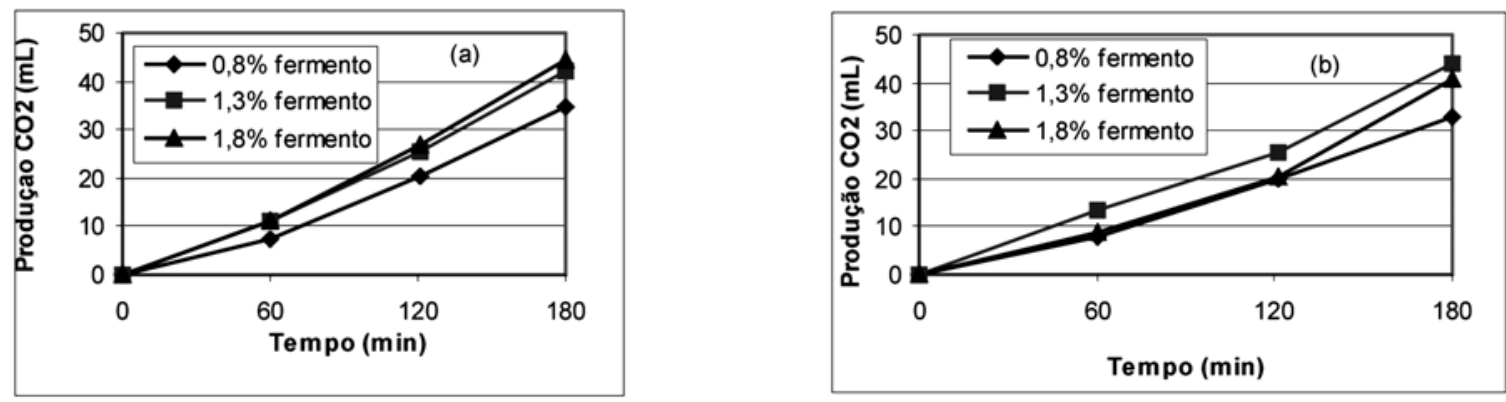

FIGURA 3 - Produção de CO2 durante a fermentação da massa congelada de pão de forma nas concentrações de 0,8\%, 1,3\% e 1,8\% de fermento após 30 dias (a) e 60 dias (b) de armazenamento

\section{3 - Escore de pontos}

Para a obtenção do escore de pontos foram avaliados volume específico, cor da crosta, quebra, simetria, crosta, cor, textura e estruturado miolo, aroma e sabor, através do uso de uma escala de pontuação. A cor da crosta dos pães de forma, elaborados a partir de massa congelada não fermentada armazenada por 30 dias e 60 dias, mostrou ser dependente das concentrações de fermento instantâneo e extrato de soja adicionadas na formulação. A concentração de lecitina de soja não interferiu significativamente $(\mathrm{p}>0,05)$ na resposta estudada.

Na Figura 4, representa-se em forma de gráficos de superficie de resposta, a variação da cor da crosta dos pães de forma em função das concentrações de fermento instantâneo e extrato de soja, nos tempos 30 dias e 60 dias de armazenamento das massas congeladas não fermentadas. A cor da crosta é uma característica subjetiva, sendo obtida a partir da avaliação de provadores em escala de zero a dez pontos. A cor da crosta aumentou com a elevação da concentração de fermento instantâneo e redução do teor de extrato de soja, tanto aos 30 dias quanto aos 60 dias de armazenamento das massas congeladas não fermentadas. Ocorreu uma redução da pontuação dada pelos provadores para cor da crosta com o aumento do periodo de armazenamento, passando de 8,25 em média, para 7,06 pontos.

RIBOTTA, LEÓN \& AÑÓN [20], em estudo do efeito do congelamento de massa na qualidade de pão, verificaram que a firmeza da crosta aumentou quando os pães elaborados a partir de massa congelada foram armazenados a $4^{\circ} \mathrm{C}$. Com a suplementação de ácido diacetil tartárico, glúten e goma guar os pães produzidos apresentaram estrutura da crosta mais aberta quando comparado com os pães produzidos com a formulação convencional. No presente trabalho foi verificada uma redução na pontuação da característica cor da crosta do pão com o aumento da concentração de extrato integral de soja ativado. De acordo com RIAZ [19], a adição de até $12 \%$ de extrato de soja em produtos de panificação requer poucas alterações no processo de panificação e, além de aumentar o teor de proteína bruta, contribui para melhorar as características do pão. Neste estudo, o máximo adicionado foi de $5 \%$, sendo observado uma redução na qualidade da cor da crosta do pão de forma.

O escore de pontos dos pães de forma elaborados a partir de massa congelada não fermentada armazenada por 30 dias e 60 dias mostrou ser dependente das concentrações de fermento instantâneo e extrato de soja adicionadas na formulação. A concentração de lecitina de so-

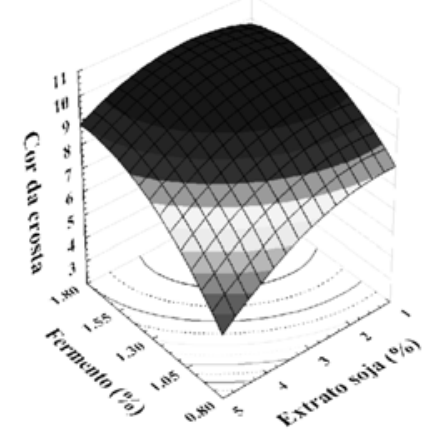

(a)

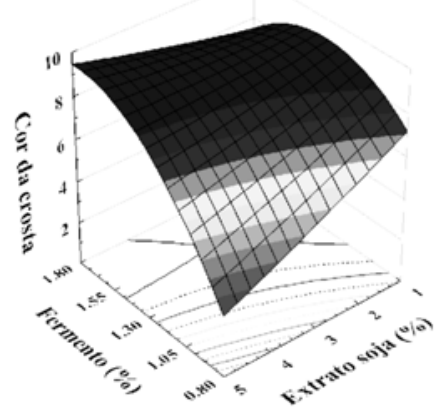

(b)

FIGURA 4 - Efeito das concentrações de fermento instantâneo e extrato de soja na cor da crosta de pão de forma produzido após 30 dias (a) e 60 dias (b) de armazenamento da massa congelada não fermentada 


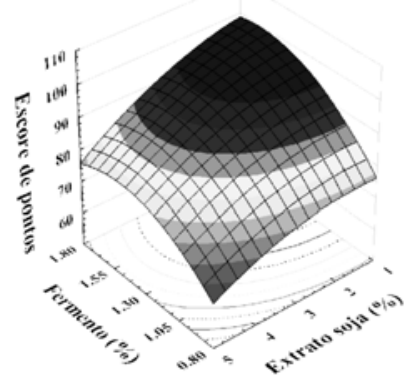

(a)

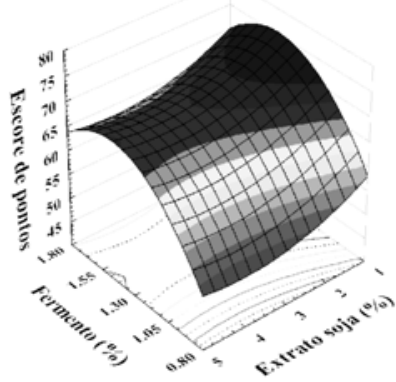

(b)

FIGURA 5 - Efeito das concentrações de fermento instantâneo e extrato de soja no escore de pontos de pão de forma produzido após 30 dias (a) e 60 dias (b) de armazenamento da massa congelada não fermentada

ja não interferiu significativamente $(p>0,05)$ na resposta estudada.

Na Figura 5, representa-se em forma de gráficos de superficie de resposta, a variação do escore de pontos dos pães de forma em função das concentrações de fermento instantâneo e extrato de soja, nos tempos 30 dias e 60 dias de armazenamento das massas congeladas não fermentadas. O escore de pontos aumentou com a elevação da concentração de fermento instantâneo e redução do teor de extrato de soja, tanto aos 30 dias, quanto aos 60 dias de armazenamento das massas congeladas não fermentadas. Ocorreu uma redução da pontuação dada pelos provadores para o escore do pão com o aumento do período de armazenamento, passando de 79,01 em média, para 60,85 pontos.

SALAS-MELLADO [23] verificou que todas as características da qualidade da massa e do pão foram prejudicadas pelo armazenamento sob congelamento por 60 dias. Das características tecnológicas do pão, a dureza foi a que apresentou as maiores mudanças no decorrer do armazenamento congelado. O indice de perda do escore de pontos aumentou em todos os tratamentos quando comparado 30 dias e 60 dias de armazenamento. Neste trabalho, o volume específico, que é uma das características avaliadas para obter o escore de pontos, não diminuiu ao longo do armazenamento. Entretanto, em todas as demais características avaliadas se verificou um prejuízo na qualidade dos pães com o aumento do tempo de armazenamento.

\section{4 - CONCLUSÕES}

Entre os ingredientes estudados na formulação de pão de forma, somente as concentrações de fermento instantâneo e de extrato de soja interferiram significativamente nas variáveis respostas. O volume e a produção de gás aumentaram com o tempo de fermentação da massa sendo proporcional à concentração de fermento utilizado na formulação. O volume específico, a cor da crosta e o escore de pontos dos pães aumentaram com a elevação da concentração de fermento instantâneo e redução do teor de extrato de soja. Com o aumento do tempo de armazenamento de 30 dias para 60 dias ocorreu uma perda nas características de qualidade dos pães, exceto para volume específico que se manteve estável.

\section{5 - REFERÊNCIAS BIBLIOGRÁFICAS}

[1] AACC- American Association of Cereal Chemists. Approved methods of the AACC. 9 ed. Saint Paul, 1995.

[2] BÁGUENA, R.; SORIANO, M. D.; MARTÍNEZANAYA, M. A; DE BARBER, C. B. Viability and performance of pure yeast strains in frozen wheat dough. Journal of Food Science, v.56, n.6, p.1690-1694, 1991.

[3] BOX, G.E.P.; BEHNKEN, D.W. Some new three level designs for the study of quantitative variables. Techonometrics, v.2, p.455-475, 1960.

[4] BRASIL. Ministério da Saúde. Agência Nacional de Vigilância Sanitária. Resolução RDC nº 90, de 18 de outubro de 2000. Aprova o regulamento técnico para fixação de identidade e qualidade de pão. Diário Oficial da República Federativa do Brasil, Brasília, outubro de 2000. Disponivel em <www.anvisa.gov.br > acesso em 26 de janeiro de 2004.

[5] BRUINSMA, B.L.; GIESENSCHLAG, J. Frozen dough performance: compressed yeast-instant dry yeast. Bakers' Digest, v.58, n.6, p.6-11, 1984.

[6] EL-DASH, A.A. Standardized mixing and fermentation procedures for experiments baking test. Cereal Chemistry, v.55, n.336, p.436-446, 1978.

[7] FERREIRA, S.M.R. Controle da qualidade em sistemas de alimentação coletiva I. São Paulo: Varela, 2002. $173 p$.

[8] FRANCISCHI, M.L., ORMENESE, R.C.S., PIZZINATTO, A. Tecnologia de produção de pães a partir de massas congeladas. Ciênc. Tecnol. Aliment., v.32, n.2, p.209214. 1998.

[9] GUTKOSKI, L.C.; JACOBSEN NETO, R. Procedimento para teste laboratorial de panificação- pão tipo forma. Ciência Rural, v.32, n.5, p.873-879, 2002.

[10] GUTKOSKI, L.C.; SANTOS, E. Estudo de formulação na 
produção de pão Francês congelado não fermentado. Revista Brasileira de Agrociência, v.10, n.3, p.347352, 2004.

[11] HAVET, M.; MANKAI, M.; LE BAIL, A. Influence of the freezing condition on the baking performance of French frozen dough. Journal of Food Engineering, v.45, p.139-145, 2000.

[12] HIRASAWA, R.; YOKOIGAWA, K.; ISOBE, Y.; KAWAI, H. Improving the freeze tolerance of baker's yeast by loading with trehalose. Bioscience, Biotechnology and Biochemistry, v. 65, n.3, p.522-526, 2001.

[13] INOUE Y.;BUSHUK, W. Studies on frozen doughs. I. Effects of frozen storage and freeze-thaw cycles on baking and rheological proprieties. Cereal Chemistry, v.56, n.5, p.627-631, 1991 .

[14] JACKEL, S. S. Frozen dough opportunities kup heating up. Cereal Foods World, v. 36, n.6, p.529, 1991.

[15] LE BAIL, A., GRINAND, C., LE CLEACH, S., MARTINEZ, S., QUILIN, E. Influence of storage conditions on frozen French bread dough. Journal of Food Engineering, v.39, p.289-291, 1999

[16] LU, W.; GRANT, L.A. Effect of prolonged storage at freezing temperatures on starch and baking quality of frozen doughs. Cereal Chemistry, v.76 n.5, p.656-662, 1999.

[17] NEMETH, L.J.; PAUlLEY, F.G.; PRESTON, K.R. Effects on ingredients and processing conditions on the frozen dough bread quality of a Canada Western Red Spring wheat flour during prolonged storage. Food Research International, v.29, n.7, p.609-616, 1996.

[18] QUAGLIA, G. Ciencia y tecnologia de la panificación. Zaragoza: Acribia, 1991.p.401-425.

[19] RIAZ, M.N. Healthy baking with soy ingredients. Cereal Foods World, v.44, n.3, p.136-139, 1999.

[20] RIBOTTA, P. D.; LEÓN, A. E.; AÑÓN, M. C. Effect of freezing and frozen storage of doughs on bread quality. Journal Agricultural Food Chemistry, v.49, p.913-918, 2001.

[21] ROUILlÉ, J.; LE BAIL, A., COURCOUX, P. Influence of formulation and mixing conditions on breadmaking qualities of French frozen dough. Journal of Food Engineering, v.43, p.197-203, 2000.

[22] SAllaS-MELladO, M.M.; CHANG, Y. K. Efeito do congelamento da massa de pão sobre as leveduras e a estrutura do glúten. Boletim SBCTA, v.33, n.2, p.194209, 1999.

[23] SALLAS-MELLADO, M.M. Estudo da influência da formulação e das condições operacionais dos tipos de congelamento na qualidade da massa e do pão. Campinas, 2003. 242p. Tese (Doutor em Tecnologia de Alimentos), Faculdade de Engenharia de Alimentos, Universidade Estadual de Campinas (UNICAMP).

[24] SILVA, R. Phospholipids as natural surfactants for the cereal industry. Cereal Foods World, v.35, n.10, p.1008-1012, 1990.

[25] SLUIMER, I.P. Principles of dough retarding. Bakers' Digest, v.55, n.4, p.6-8, 10, 1981.

[26] STAUFFER, C.E. Frozen bakery products. IN: MALLETT, C.P. (ed) Frozen Food Tecnology. Cambridge: Chapman \& Hall, 1994.

[27] TWEED, A.R. A look at French "French Bread". Cereal Foods World, v.28, n.27, p.397-399, 1983.

[28] WANG, Z.J.; PONTE, J.G.Jr. Improving frozen dough qualities with the addition of vital wheat gluten. Cereal Foods World, v.39, n.7, p.500-503, 1994.

[29] WATANABE, E; BENASSI, V. T., O uso de massa congelada na produção de pão. Boletim CEPPA, v.18, n. 1, p.71-84, 2000.

\section{6 - AGRADECIMENTOS}

Os autores agradecem à Fundação de Amparo à Pesquisa do Estado do Rio Grande do Sul (FAPERGS) pelo auxílio financeiro ao projeto. À FAPERGS e ao CNPq pelas bolsas de iniciação científica. Às empresas Olvebra Industrial, Solae do Brasil, Granotec e Bruggeman, pelo fornecimento de ingredientes utilizados nos experimentos. 